\title{
Exploring Repurposing Potential of Existing Drugs in the Management of COVID-19 Epidemic: A Critical Review
}

\author{
Debjit Chakraborty $^{\mathrm{a}}$, Falguni Debnath ${ }^{\mathrm{a}, \mathrm{c}}$, Subrata Biswas ${ }^{\mathrm{a}}$, Mihir Bhatta ${ }^{\mathrm{a}}$, Suman Ganguly ${ }^{\mathrm{b}}$, \\ Alok Kumar Deb ${ }^{\text {a }}$, Malay Kumar Saha ${ }^{\text {a }}$, Shanta Dutta ${ }^{\text {a }}$
}

\begin{abstract}
Despite high morbidity and mortality of ongoing coronavirus disease 2019 (COVID-19) pandemic, no specific therapy has been established till date. Though in vitro studies identified various molecules as possible therapies against severe acute respiratory syndrome coronavirus 2 (SARS-CoV-2), these findings call for substantiation by human studies. We conducted this review aiming at reporting evidences on therapies used so far globally for management of COVID-19 in clinical settings. We searched electronic databases as PubMed, Google Scholar, EMBASE and extracted 612 possible studies as on May 31, 2020. We included original studies of any epidemiological design done on human COVID-19 patients and measured clinical outcomes. Finally, following removal of duplicates and studies meeting exclusion criteria, we derived 22 studies, of which eight were clinical trials, seven were case reports and case series, and seven were observational studies. The most reported therapies were hydroxychloroquine (HCQ) (eight studies) and lopinavir/ritonavir (four studies). We conclude from the evidence generated so far that interferon combined with antivirals, remdesivir, umifenovir and favipiravir were mostly associated with better clinical outcomes. The therapeutic effect of HCQ was established initially by two clinical trials; one of them showing a reinforcing effect by azithromycin but subsequent studies did not elicit any effectiveness rather increased rate of adverse events was reported. Lopinavir/ritonavir was found beneficial when administered with interferon and ribavirin, but one clinical trial on its sole use proved contrary. As many clinical trials are in process, we expect to get concrete evidences on repurposing of existing drugs based on less biased, high powered studies.
\end{abstract}

Keywords: COVID-19; Repurposing; Remdesivir; HCQ

Manuscript submitted May 29, 2020, accepted June 12, 2020

Published online July 22, 2020

aICMR-National Institute of Cholera and Enteric Disease, Kolkata, India ${ }^{b}$ West Bengal State AIDS Prevention and Control Society, West Bengal, India ${ }^{\mathrm{C} C}$ Corresponding Author: Falguni Debnath, Division of Epidemiology, ICMRNational Institute of Cholera and Enteric Disease, P-33, Scheme XM, CIT Road, Beliaghata, Kolkata 700010, West Bengal, India.

Email: falgunidebnath@yahoo.in

doi: https://doi.org/10.14740/jocmr4241

\section{Introduction}

Coronavirus disease 2019 (COVID-19) epidemic though initiated in Wuhan City of China in December 2019, has now changed in to global pandemic affecting 213 countries. Number of reported cases and death worldwide amounts to 3,349,786 cases and 238,628 (as on May 3, 2020), thus a case fatality rate (CFR) as high as 7.1\% [1]. European and American regions are worst hit. Though $80 \%$ of all severe acute respiratory syndrome coronavirus 2 (SARS-CoV-2) infection cases are asymptomatic or having milder symptoms, severity has been observed to be increased with age and presence of comorbidity. In China, CFR is $8.0 \%$ and $14.8 \%$ in people aged $70-79$ years and $\geq 80$ years, respectively as compared to overall CFR of $2.3 \%$ [2]. A meta-analysis involving 19 studies (18 from China and one from Australia) reported, fever $(88.7 \%, 95 \%$ confidence interval $(\mathrm{CI}): 84.5-92.9 \%)$, cough $(57.6 \%, 95 \%$ CI: $40.8-74.4 \%)$ and dyspnea (45.6\%, 95\% CI: $10.9-80.4 \%)$ as the most prevalent clinical manifestations. Whereas, severe outcomes such as acute respiratory distress syndrome (ARDS) (32.8\%, 95\% CI: 13.7 - 51.8), cardiac injury $(13.0 \%, 95 \%$ CI: $4.1-21.9 \%)$, acute renal injury (7.9\%, 95\% CI: $1.8-14.0 \%)$, shock $(6.2 \%, 95 \%$ CI: 3.1-9.3\%) were reported. Comorbidities were reported in $36.8 \%$ of cases (95\% CI: $24.7-48.9 \%$ ), frequent being hypertension (18.6\%, 95\% CI: 8.1-29.0\%), cardiovascular disease $(14.4 \%, 95 \%$ CI: 5.7-23.1\%), and diabetes (11.9\%, 95\% CI: 9.1-14.6\%) [3].

In absence of any specific antiviral drug, treatment till date is symptomatic and supportive. In severe cases, intravenous (IV) fluid, oxygen inhalation, ventilator supports are the available options at present $[4,5]$. Owing to very high transmission rate and higher CFR among elderly and patients with underlying comorbidity, it is imperative to explore possible options from the existing drugs which can reduce severity to some extent and thus mortality can be minimized. Currently, investigations are ongoing globally to understand whether molecules approved earlier for treatment of other viral diseases can be used effectively in the management of COVID-19, either as therapy or prophylaxis [6]. Many countries have initiated practice of available drugs with limited evidence as empirical treatment. In India, hydroxychloroquine (HCQ) (dose: $400 \mathrm{mg}$ bis in die (BID) for 1 day followed by $200 \mathrm{mg}$ BID for 4 days) combined with azithromycin (AZ) (500 mg quaque die (QD) for 5 days) has been considered as an off-label indication in patients with severe disease and requiring intensive care management [7]. 


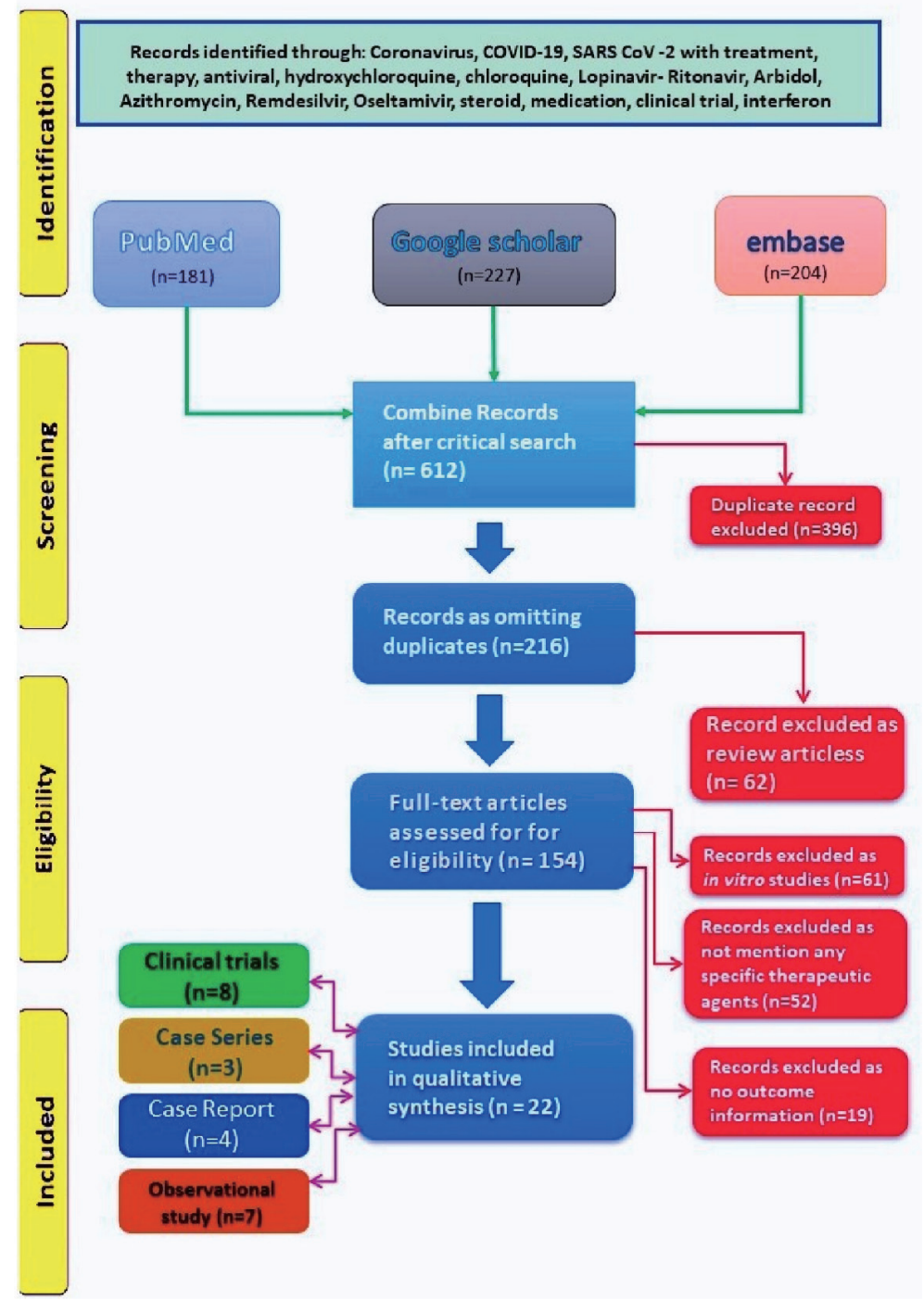

Figure 1. PRISMA diagram showing literature search and stepwise derivation to eligible papers. PRISMA: preferred reporting items for systematic reviews and meta-analyses.

Hence, in this review we explored the existing literatures on available therapies of COVID -19 to collate evidence on possibility of repurposing the existing drugs for management of COVID-19 cases with some effectiveness.

\section{Literature Search}

We searched for published literature in PubMed, Google Scholar and EMBASE with the following search keys: coronavirus/COVID-19/SARS CoV-2 with treatment/therapy/antiviral/hydroxychloroquine/chloroquine/lopinavir-ritonavir/Arbidol/azithromycin/remdesilvir/oseltamivir/steroid/medication/clinical trial/ interferon.

We included the studies in analysis which met the following criteria: 1) In vivo studies, i.e., on confirmed COVID-19 human cases; 2) Original articles (clinical trial, case series, and case reports, etc.); 3) Where a specific existing therapeutic agent (drug) along with some outcome (clinical or virological) is mentioned.

We excluded the papers which: 1) Were based on in vitro studies, i.e., preclinical or animal model based; 2) Were review articles/commentary; 3) Did not mention any specific existing drug; 4) Mentioned use of a drug without any patient outcome information.

We obtained 612 papers from combined search of three databases as on May 31, 2020. Following elimination of duplicates (396) we derived at 216 articles. After removing the articles as per exclusion criteria, we retained 22 articles for the review (Fig. 1).

\section{Literature Retrieved}

The distribution of 22 studies under assessment, both drug 
Table 1. Distribution of 22 Studies Under Assessment, Both Drug Wise and Study Design Wise

\begin{tabular}{lllll}
\hline \multirow{2}{*}{ Drugs/therapy } & \multicolumn{3}{c}{ Study design } \\
\cline { 2 - 4 } & Clinical trial & Case series & Case report & Observational study \\
\hline HCQ/CQ with macrolide (azithromycin/clarithromycin) & 1 & 1 & 1 & 2 \\
HCQ & 2 & 1 & 1 & 2 \\
Lopinavir/ritonavir & 1 & & 4 \\
Umifenovir/favipiravir & 1 & 1 & 1 & 1 \\
Methylprednisolone (with other agent) & & 1 & 1 \\
Thalidomide with methylprednisolone & & & 1 \\
Remdesivir & 2 & 1 & 3 \\
Interferon (in combination) & 1 & 3 & 4 & 7 \\
Total & 8 & & 3 \\
\hline
\end{tabular}

$\mathrm{HCQ}$ : hydroxychloroquine; $\mathrm{CQ}$ : chloroquine.

wise and study design wise was shown in Table 1 . We observed that HCQ (eight studies) and lopinavir/ritonavir (four studies) were discussed maximum. In three of the eight papers for the former, macrolides such as AZ/clarithromycin was added with HCQ. Methylprednisolone was discussed in three papers, though in two of them it was given along with immunoglobulin and in one study, it was administered along with thalidomide. Remdesivir and interferon (IFN) were discussed in three papers each. All the studies were on virologically confirmed (reverse transcription polymerase chain reaction (RT-PCR) of nasopharyngeal/throat swab) COVID-19 cases. Among the 22 studies, 14 were from China, two each from France and USA, one each from Hong Kong and Columbia, remaining two studies were based on multinational cohort analysis. Eleven of them were based on single centre/hospital, and the rest 11 studies were multicentric.

\section{Description of each therapeutic agent}

We have summarized the major variables of each article in Supplementary Material 1 (www.jocmr.org). We discussed the studies as per each drug/agent along with their related therapeutic properties.

\section{HCQ/AZ}

The open-label non-randomized clinical trial conducted [8] in two hospitals of France revealed that HCQ treatment was significantly effective in COVID-19 patients and its effect was reinforced by AZ. Oral HCQ sulfate $200 \mathrm{mg}$, three times daily for 10 days along with $\mathrm{AZ}$ at $500 \mathrm{mg}$ on first day followed by $250 \mathrm{mg}$ per day for next 4 days was given to the PCRconfirmed COVID-19 cases aged $>12$ years in the intervention arm. At the sixth day post-inclusion, all patients treated with HCQ and AZ combination were virologically cured as compared to $57.1 \%$ in patients treated with HCQ only, and $12.5 \%$ in the control group $(\mathrm{P}<0.001)$. Though this was a clinical trial providing the evidence of effectiveness, it had two major limitations: 1) Non-randomization; and 2) Inadequate sample size (total 36, intervention arm: 20, and control arm: 16). Hence, further validation with robustness may be necessary. Another randomized control trial [9] of HCQ alone from China on 62 patients (31 in each arm) reported significantly shortening of body temperature recovery time and cough remission time in HCQ treatment group. Improvement rate of pneumonia in HCQ $\operatorname{arm}(80.6 \%, 25$ of 31$)$ was much higher than control $\operatorname{arm}(54.8 \%, 17$ of 31$)$. However, this publication was not peer reviewed. A case series of 100 patients from 10 hospitals of China showed that the treatment with chloroquine (CQ) phosphate was effective in averting exacerbations of pneumonia, minimizing lung involvements in radioimaging, accelerating a virus-negative conversion, thereby shortening the disease course and severity, without any adverse effect at therapeutic dose [10].

Further studies [11-14], including a multicenter, openlabel, randomized controlled trial [12] on HCQ with or without macrolide from different nations did not elicit any significant benefit over standard care in terms of intubation, mortality, survival, and negative virological outcome. Proportion of comorbid conditions such as hypertension, diabetes, and chronic lung diseases, etc., were more or less similar across HCQ and non-HCQ groups in two of these studies [12, 13]; however HCQ was administered to the patients with higher frequency of comorbidities in remaining two studies $[11,14]$. In all of them, higher proportion of patients in HCQ groups reported adverse effects like arrhythmia, cardiac arrest, other electrocardiogram (ECG) changes and diarrhea. A case report from Columbia showed clinical and virological improvement with the use of CQ + clarithromycin combination though the case is a 34 years old patient with no major comorbidity [15]. It is pertinent to mention the studies showing beneficial effect with HCQ included patients with relatively lower mean age (44 - 46 years) compared to those showing no therapeutic benefit (mean age: 60 years and above). Hence age of the patient confounding the study outcome might be a possibility.

CQ and its derivative HCQ are aminoquinolinic compounds used for the treatment of malaria and autoimmune 
diseases like rheumatoid arthritis and systemic lupus erythematosus (SLE) [16]. Data on anti-viral potential of CQ were generated from experiments on human immunodeficiency virus (HIV), hepatitis A, and influenza viruses [17].

In February 2020, the evidence of in vitro efficacy of CQ on SARS-CoV-2 was reported with an effective concentration (EC) 90 value of $6.90 \mu \mathrm{M}$, achievable with clinically used dosing [18]. These observations were subsequently corroborated by other authors based on their in vitro studies on anti-viral activity of CQ and HCQ. HCQ showed more potent than CQ with lower EC 50 values, possibly due to slow and progressive accumulation of HCQ at higher concentrations into the cells [19].

\section{Lopinavir/ritonavir}

In the randomized, controlled, open-label trial [20], lopinavir/ritonavir (400 $\mathrm{mg}$ and $100 \mathrm{mg}$ ) was administered twice a day for 14 days on 94 severe adult patients of confirmed COVID-19 (100 cases in control arm received standard care only) in a single centre of Wuhan, China. The study did not elicit any significant benefit with lopinavir/ritonavir treatment beyond standard care (hazard ratio for median time to clinical improvement: 1.39; 95\% CI: 1.00 - 1.91). A case report from Korea with a recent travel history from Wuhan showed reduction in viral load following administration of two tablets (lopinavir $200 \mathrm{mg} /$ ritonavir $50 \mathrm{mg}$ ) twice daily [21]. In a retrospective observational study, following use of lopinavir, viral load, radiography and eosinophil count improved continuously among 10 patients, although the patients were also given IFN$\alpha 2 b$ atomization inhalation, Arbidol, methylprednisolone and immunoglobulin [22]. Hence, evidence generated from the last two studies was very weak due to lack of control arm, presence of confounders and small sample size. Another single centre retrospective observational study on 78 patients from China treated with lopinavir/ritonavir reported a beneficial effect in terms of significant shortening of duration of viral shredding among patients initiating treatment within 10 days of onset of symptom compared with those without lopinavir/ritonavir treatment (median: 19 days vs. 28.5 days, log-rank $\mathrm{P}<0.001$ ) [23].

Lopinavir and ritonavir are primarily protease inhibitors, used in combination as antiretroviral treatment against HIV. When combined with lopinavir the main drug, ritonavir produces a pharmacological boosting by inhibiting cytochrome P450-3A4 enzyme responsible for metabolism of lopinavir, thereby prolonging its bioavailability and plasma half life [24]. Lopinavir was identified as having inhibitory action against human SARS-CoV and Middle East respiratory syndrome coronavirus (MERS-CoV) by in vitro studies [25-27].

\section{Methylprednisolone in combination}

Methylprednisolone, a steroid was discussed in combination with other therapeutic agents in two case reports and one case series [28-30]. In the case series of 10 patients, short-term moderate-dose corticosteroid (160 mg/day) plus immunoglobulin (20 g/day) was found to reverse the disease progression in $90 \%$ patients in whom condition worsened earlier with low-dose steroid therapy. APACHE II score $(9.10 \pm 6.15$ vs. $5.50 \pm 9.01, \mathrm{P}<0.05)$, temperature $(37.59 \pm 1.16$ vs. 36.46 $\pm 0.25, \mathrm{P}<0.05)$, lymphocyte count $(0.59 \pm 0.18$ vs. $1.36 \pm$ $0.51, \mathrm{P}<0.05)$, serum lactate dehydrogenase $(419.24 \pm 251.31$ vs. $257.40 \pm 177.88, \mathrm{P}<0.05)$, and $\mathrm{C}$-reactive protein (49.94 \pm 26.21 vs. $14.58 \pm 15.25, \mathrm{P}<0.05)$ significantly improved in moderate-dose as compared with low-dose therapy [28]. Methylprednisolone was mentioned to be administered in two case reports; in one with combination of Arbidol, oseltamivir and moxifloxacin [29], and in another, with thalidomide [30]. Though clinical improvement was noted in both the case reports, but the number of cases were very low (2 and 1). Moreover, as used in combination, it was difficult to ensure the major contributory agent. Thalidomide was given with methylprednisolone in a patient of COVID-19 confirmed pneumonia and subsequently increased oxygenation index was reported along with other clinical improvement. Though methylprednisolone and thalidomide do not have any proven virucidal role, still they are used to reduce complications owing to their anti-inflammatory and immunomodulatory effect and thereby aiding into clinical improvement. In absence of any specific targeted therapy, their role may also be perceived as important in terms of overall clinical improvement.

\section{Umifenovir (Arbidol) vs. favipiravir}

A prospective, randomized, controlled, open-label multicenter trial compared the efficacy of umifenovir (Arbidol) vs. favipiravir among 240 (120 each drug) adult cases of confirmed COVID-19. Each patient received either umifenovir (Arbidol) as $200 \mathrm{mg}$ thrice daily or favipiravir $1,600 \mathrm{mg}$ twice daily on the first day followed by $600 \mathrm{mg}$ twice daily for 10 days. At day 7 of follow-up, clinical recovery rate did not differ significantly between favipiravir group $(61.2 \%)$ and Arbidol group $(51.6 \%)$. Favipiravir was associated with shorter latencies for recovery from fever (difference: 1.70 days, $\mathrm{P}<0.0001$ ) and cough (difference: 1.75 days, $\mathrm{P}<0.0001$ ). Rise in the level of serum uric acid was also reported in the favipiravir group. Otherwise both the drugs were found to be similarly effective so far and a clinical decision making can be done optimizing the patient's disease status and comorbidity while selecting any one among the two [31]. Both the molecules act as inhibitor of RNA-dependent RNA polymerase (RdRP) and approved for treatment of influenza in Russia, China (umifenovir) and Japan (favipiravir) [32-36].

\section{Remdesivir}

The multinational (USA, Canada, Europe and Japan) cohort analysis on compassionate use of 10-day course of remdesivir [37] on 53 SARS-CoV-2 confirmed patients on oxygen support or with $\mathrm{O}_{2}$ saturation $\leq 94 \%$ in ambient air revealed $84 \%$ clinical improvement at 28 days follow-up (95\% CI: 77- 99\%). 
Clinical improvement was defined by either a decrease of two points or more on the six-point ordinal scale or live discharge. Over a median follow-up of 18 days after the first dose $68 \%$ (36) reported improvement in category of oxygen support while condition deteriorated in $15 \%$ (8) cases. Improvement was evident in all patients (12) breathing ambient air or receiving low-flow supplemental oxygen and in $71 \%(5 / 7)$ of those receiving noninvasive oxygen support. During follow-up 60\% patients reported adverse events such as raised liver enzymes, diarrhea, rash, renal compromise and hypotension. Serious adverse events like multi-organ dysfunction, shock, acute kidney injury were found among $23 \%$ cases.

One multicentric randomized, double-blind, placebo-controlled trial from China on 237 confirmed COVID-19 adult patients did not elicit significant difference in time to clinical improvement (hazard ratio: 1.23, 95\% CI: 0.87 - 1.75) following use of remdesivir for 10 days as compared to control group. Sixty-six percent and $18 \%$ patients reported adverse and serious adverse events respectively such as constipation, hypoalbuminemia, hypokalemia, anemia, thrombocytopenia, raised total bilirubin. Due to non-availability of required sample size the study could achieve only a power of $58 \%$, hence the results needs to be interpreted with caution [38].

The recently published [39] randomized, double-blind, placebo-controlled, multicentre trial on 1,059 patients (538 in remdesivir and 521 in placebo arm) from multiple continents showed a therapeutic effectiveness in the remdesivir group in terms of shorter median time to recovery as compared to placebo group (11 vs. 15 days) (relative risk (RR): 1.32; 95\% CI: $1.12-1.55 ; \mathrm{P}<0.001)$. Mean age of this study (58 years) was not much lower than that of previous two (64 - 65 years), still the drug was found to be therapeutically effective although around $30 \%$ receiving the drug reported one or more adverse events which may be a concern during evidence to policy translation at large scale.

Remdesivir is a prodrug of a nucleotide analogue and intracellularly metabolized to an analogue of adenosine triphosphate that inhibits viral RNA polymerases. Remdesivir is a broad-spectrum antiviral active against various virus families, namely filoviruses (e.g., Ebola) and coronaviruses (e.g., SARS-CoV and MERS-CoV). Its prophylactic and therapeutic efficacy against coronaviruses was reported in preclinical models [18, 40-42]. In vitro experiments reported its activity against SARS-CoV-2. The safety profile of the drug was also established based on its use on Ebola infected patients [43, 44].

The former study generated evidence of overall clinical improvement with application of remdesivir in severe cases of COVID-19 in multiple continents. Though there is no control arm in the study however different parameters for analysis present in this study such as six-point ordinal scale, oxygenation category, discharge rate, adverse event had thrown light towards its effective potential; however the later clinical trial failed to elicit any significant clinical benefit. This inconsistency in observation and particularly the lack of statistical power in the clinical trial call for further exploration and validation by clinical trial with adequate sample size. At present in the dearth of concrete evidence from human subject, there will remain enough room for ambiguity, dilemma and subjective variation for clinical decision making.

\section{IFN in combination}

IFN- $\alpha 2$ b was administered along with other antivirals in two studies. In a retrospective study of 77 patients from single centre of China [45], IFN- $\alpha 2 b$ as nebulized aerosol was given either alone (seven patients) and with umifenovir (Arbidol) (46 patients) as compared to umifenovir alone ( 24 patients). IFN- $\alpha 2 b$ with or without Arbidol was observed to reduce significantly the duration of detectable viral load in the upper respiratory tract specimen as compared to umifenovir alone. Also the duration of elevated blood levels for interleukin-6 and C-reactive protein was reduced in the IFN arms. Hence it appears that addition of IFN was effective in promoting virological recovery and minimizing cytokine surge. However, it is pertinent to mention that mean age of umifenovir alone group (64.5 years) was much higher ( $\mathrm{P}<0.01)$ as compared to IFN groups (40 - 41 years), which might have any influence in overall clinical outcome.

The recently published evidence from multicentric, prospective, open-label, randomized, phase II trial from Hong Kong where IFN- $\beta 1$ b was used in combination with lopinavir/ ritonavir and ribavirin against the control arm of lopinavir/ritonavir alone [46]. A total of 127 patients were recruited (86 in combination group and 41 in the control group). The median number of days from onset of symptom to start of treatment was 5 days (interquartile range (IQR): 3 - 7). Median duration from initiation of treatment to negative RT-PCR of nasopharyngeal swab was significantly shorter in the combination group (7 days, IQR: 5 - 11) than control group (12 days (8 - 15); hazard ratio: $4.37,95 \% \mathrm{CI}: 1.86-10.24, \mathrm{P}=0.0010)$. Adverse events were minimal (self-limited nausea and diarrhoea) without any difference between two groups. Another multicentric case series on 18 patients from China treated with IFN-2b in combination with lopinavir/ritonavir showed clinical improvement in many domains though median age of the group was only 41 years (IQR: 31 - 51 years) [47]. All the above studies showed some benefit of IFN + antiviral combination treatment though the median/mean ages in these studies were relatively lesser as compared to studies conducted for other drugs.

IFNs are low molecular weight proteins produced by infected cells following viral pathogenesis and constitute the first line of defense against infections [48]. The expression of the IFNstimulated genes (ISGs) is induced by IFN, in the infected as well as in neighboring cells. These lead to development of an antiviral environment, thus inhibiting further viral replication [49, 50]. The IFNs augments the immune system in various ways, resulting in different antiviral, antiproliferative and immunomodulatory activities [48] when used with other antivirals like remdesivir and ribavirin, which was reported to be effective against MERS$\mathrm{CoV}$ in both in vitro and in vivo $[42,51]$ studies. We observed improvement in terms of viral clearance with the use of IFN molecules compared to control arm consistently in both the studies.

\section{Other drugs}

Oseltamivir has been found effective against H1N1 influenza and tried empirically in some of the studies of COVID-19 along with other compounds. However, any conclusive outcomes of 
Table 2. Mechanism of Action of Drug Candidates on SARS-CoV-2 Infection

\begin{tabular}{|c|c|c|}
\hline Drugs & Types & Mechanism of action \\
\hline $\begin{array}{l}\text { Chloroquine and } \\
\text { hydroxychloroquine }\end{array}$ & 4-aminoquinoline & The post-translation alteration of newly synthesized proteins via glycosylation inhibition [52]. \\
\hline Umifenovir & Fusion inhibitor & Viral fusion inhibition with the targeted membrane, which blocks virus entry into the cell [54]. \\
\hline Favipiravir & $\begin{array}{l}\text { RNA polymerase } \\
\text { inhibitors }\end{array}$ & $\begin{array}{l}\text { Favipiravir is a prodrug that is ribosylated and phosphorylated intracellularly to form the active } \\
\text { metabolite favipiravir ibofuranosyl-5'-triphosphate (T-705-RTP). T-705-RTP competes with } \\
\text { purine nucleosides and interferes with viral replication by incorporation into the virus RNA and } \\
\text { thus, potentially inhibiting the RNA-dependent RNA polymerase (RdRP) of RNA viruses [31]. }\end{array}$ \\
\hline Methylprednisolone & Corticosteroids & $\begin{array}{l}\text { Prolongs the survival time and prevents complication of clinical cases } \\
\text { through its anti-inflammatory and immunomodulatory action [55]. }\end{array}$ \\
\hline Thalidomide & Immunomodulators & $\begin{array}{l}\text { It has an anti-inflammatory action due to its ability to speed up the degradation of messenger } \\
\text { RNA in blood cells and thus reduce tumor necrosis factor- } \alpha \text { (TNF- } \alpha \text { ). Furthermore, thalidomide } \\
\text { can increase the secretion of interleukins (IL), such as IL-12, and activate natural killer cells [56]. }\end{array}$ \\
\hline Interferon & $\begin{array}{l}\text { Low molecular } \\
\text { weight protein }\end{array}$ & $\begin{array}{l}\text { The expression of the interferon-stimulated genes (ISGs) is induced by interferon, in the infected } \\
\text { as well as in neighboring cells. These lead to development of an antiviral environment, thus } \\
\text { inhibiting further viral replication. The interferons augment the immune system in various ways, } \\
\text { resulting in different antiviral, antiproliferative and immunomodulatory activities [48-50]. }\end{array}$ \\
\hline
\end{tabular}

SARS-CoV-2: severe acute respiratory syndrome coronavirus 2.

oseltamivir on SARS-CoV-2 from clinical studies are yet to be reported. Drugs like teicoplanin and ivermectin are also under exploration based on their possible antiviral properties in vitro.

Mechanism of the drug candidates on SARS-CoV-2 infection were summarized in Table 2 [31, 37, 48-50, 52-56].

\section{Therapeutic Dilemma}

The drugs administered in different trials and in empirical mode were actually based on their experience on MERS-CoV. Since most of the times combination therapies were used and comparison were made among their effectiveness, it is very difficult to comment on which may be used as main therapeutic agents. One or more compound might have different action in the combination such as confounder, effect modifier, synergy, pharmacological boosting, etc. Using multiple drugs always carry possibilities of drug interaction, adverse reaction, and low compliance. Inconsistencies in the ultimate inference on effectiveness of one drug across different studies owing to different combination, study design, inclusion criteria, age and sex distribution, sample size, etc., create more and more dilemma to the clinicians in taking therapeutic decisions. A standardized treatment protocol would have been the ideal but looks a far reality at present.

\section{Conclusions}

We conclude from the evidence analyzed so far that the therapeutic effect of HCQ has been established initially by at least two clinical trials, and one of these showed reinforcing effect of AZ along with HCQ. However subsequent studies including one clinical trial did not elicit any therapeutic benefit rather reported increased rate of adverse event. The therapeutic combination of IFN with antivirals proved to be consistently effective across three studies. Among antivirals, remdesivir was found effective in one of the two randomized, double-blind, placebo-controlled, multicentre trial and in a cohort study. Umifenovir (Arbidol) and favipiravir showed some therapeutic effect, based on a single clinical trial, the possible repurposing may be thought of among these few drugs based on clinical conditions of the patient. Though case reports and observation studies showed some benefit of lopinavir/ritonavir, however the sole randomized, controlled, open-label trial reported the contrary. As many clinical trials are in process, we expect to get evidences on the possibility of repurposing of existing drug based on robust design, less biased, high powered, precise studies. In near future the Multi-country Solidarity Trial launched by the World Health Organization (WHO) and its partner nations will lead the globe by exploring the effectiveness of four treatment options: 1) remdesivir; 2) CQ/HCQ; 3) lopinavir with ritonavir; and 4) lopinavir with ritonavir plus IFN- $\beta 1$ a. Enrolling patients in many nations, the Solidarity Trial aims at generating concrete evidence of slowing disease progression or improving survival by any of these drugs [57].

\section{Limitations}

We have to limit our endeavor to a critical review only. We could not attempt a meta-analysis exercise since the studies on 
each drug are very less in number and hardly comparable in terms of study design and outcome variable. Most of the studies have biases like confounders, lack of randomization, and absence of control group, etc.

\section{Supplementary Material}

Suppl 1. Synopsis of 22 Papers Under Review.

\section{Acknowledgments}

None to declare.

\section{Financial Disclosure}

None to declare.

\section{Conflict of Interest}

None to declared.

\section{Author Contributions}

DC: conceptualization, literature search, analysis, interpretation, writing first draft, reviewed and finalized the manuscript. FD: conceptualization, literature search, analysis, interpretation, reviewed and finalized the manuscript. SB: literature search, analysis, reviewed and finalized the manuscript. MB: literature search, analysis, reviewed and finalized the manuscript. SG: literature search, analysis, reviewed and finalized the manuscript. AKD: conceptualization, analysis, interpretation, reviewed and finalized the manuscript. MKS: conceptualization, administrative support, interpretation, reviewed and finalized the manuscript. SD: conceptualization, administrative support, interpretation, reviewed and finalized the manuscript.

\section{Data Availability}

The authors declare that data supporting the findings of this study are available within the article.

\section{References}

1. World Health organization. Coronavirus disease (COVID-19), Situation Report-104. May 3, 2020. https:// www.who.int/docs/default-source/coronaviruse/situation-reports/20200503-covid-19-sitrep-104.pdf?sfvrsn= 53328f46_2. Last accessed: May 4, 2020.

2. $\mathrm{Wu} \mathrm{Z}, \mathrm{Mc}$ Googan JM. Characteristics of and important lessons from the coronavirus disease 2019 (COVID-19) outbreak in China: summary of a report of 72314 cases from the chinese center for disease control and prevention. JAMA. 2020.

3. Rodriguez-Morales AJ, Cardona-Ospina JA, Gutierrez-Ocampo E, Villamizar-Pena R, Holguin-Rivera Y, Escalera-Antezana JP, Alvarado-Arnez LE, et al. Clinical, laboratory and imaging features of COVID-19: A systematic review and meta-analysis. Travel Med Infect Dis. 2020;34:101623.

4. Prevention \& Treatment. US Centers for Disease Control and Prevention. February 15, 2020. Archived from the original on December 15, 2019. Retrieved January 21, 2020.

5. Overview of novel coronavirus (2019-nCoV) - Summary of relevant conditions. BMJ. Archived from the original on January 31, 2020. Retrieved February 1, 2020.

6. McCreary EK, Pogue JM. Coronavirus disease 2019 treatment: a review of early and emerging options. Open Forum Infect Dis. 2020;7(4):ofaa105.

7. https://www.mohfw.gov.in/pdf/RevisedNationalClinicalManagementGuidelineforCOVID1931032020.pdf.

8. Gautret P, Lagier JC, Parola P, Hoang VT, Meddeb L, Mailhe M, Doudier B, et al. Hydroxychloroquine and azithromycin as a treatment of COVID-19: results of an open-label non-randomized clinical trial. Int J Antimicrob Agents. 2020:105949.

9. Chen ZW, Hu JJ, Zhang ZW, Jiang S, Han SM, Yan DD, Zhuang RH, et al. Efficacy of hydroxychloroquine in patients with COVID-19: results of a randomized clinical trial. medRxiv. 2020.03.22.20040758.

10. Gao J, Tian Z, Yang X. Breakthrough: Chloroquine phosphate has shown apparent efficacy in treatment of COVID-19 associated pneumonia in clinical studies. Biosci Trends. 2020;14(1):72-73.

11. Geleris J, Sun Y, Platt J, Zucker J, Baldwin M, Hripcsak G, Labella A, et al. Observational study of hydroxychloroquine in hospitalized patients with Covid-19. N Engl J Med. 2020;382(25):2411-2418.

12. Tang W, Cao Z, Han M, Wang Z, Chen J, Sun W, Wu Y, et al. Hydroxychloroquine in patients with mainly mild to moderate coronavirus disease 2019: open label, randomised controlled trial. BMJ. 2020;369:m1849.

13. Mahevas M, Tran VT, Roumier M, Chabrol A, Paule R, Guillaud C, Fois E, et al. Clinical efficacy of hydroxychloroquine in patients with covid-19 pneumonia who require oxygen: observational comparative study using routine care data. BMJ. 2020;369:m1844.

14. Rosenberg ES, Dufort EM, Udo T, Wilberschied LA, Kumar J, Tesoriero J, Weinberg P, et al. Association of treatment with hydroxychloroquine or azithromycin with in-hospital mortality in patients with COVID-19 in New York State. JAMA. 2020.

15. Millan-Onate J, Millan W, Mendoza LA, Sanchez CG, Fernandez-Suarez H, Bonilla-Aldana DK, RodriguezMorales AJ. Successful recovery of COVID-19 pneumonia in a patient from Colombia after receiving chloroquine and clarithromycin. Ann Clin Microbiol Antimicrob. 2020;19(1):16.

16. Shukla AM, Wagle Shukla A. Expanding horizons for clinical applications of chloroquine, hydroxychloroquine, 
and related structural analogues. Drugs Context. 2019;8.

17. Savarino A, Boelaert JR, Cassone A, Majori G, Cauda R. Effects of chloroquine on viral infections: an old drug against today's diseases? Lancet Infect Dis. 2003;3(11):722-727.

18. Wang M, Cao R, Zhang L, Yang X, Liu J, Xu M, Shi Z, et al. Remdesivir and chloroquine effectively inhibit the recently emerged novel coronavirus $(2019-\mathrm{nCoV})$ in vitro. Cell Res. 2020;30(3):269-271.

19. Sarma P, Kaur H, Kumar H, Mahendru D, Avti P, Bhattacharyya A, Prajapat M, et al. Virological and clinical cure in COVID-19 patients treated with hydroxychloroquine: A systematic review and meta-analysis. J Med Virol. 2020;92(7):776-785.

20. Cao B, Wang Y, Wen D, Liu W, Wang J, Fan G, Ruan L, et al. A Trial of lopinavir-ritonavir in adults hospitalized with severe COVID-19. N Engl J Med. 2020;382(19):17871799.

21. Lim J, Jeon S, Shin HY, Kim MJ, Seong YM, Lee WJ, Choe KW, et al. Case of the index patient who caused tertiary transmission of COVID-19 infection in Korea: the application of lopinavir/ritonavir for the treatment of COVID-19 infected pneumonia monitored by quantitative RT-PCR. J Korean Med Sci. 2020;35(6):e79.

22. Liu F, Xu A, Zhang Y, Xuan W, Yan T, Pan K, Yu W, et al. Patients of COVID-19 may benefit from sustained Lopinavir-combined regimen and the increase of Eosinophil may predict the outcome of COVID-19 progression. Int J Infect Dis. 2020;95:183-191.

23. Yan D, Liu XY, Zhu YN, Huang L, Dan BT, Zhang GJ, Gao YH. Factors associated with prolonged viral shedding and impact of Lopinavir/Ritonavir treatment in hospitalised non-critically ill patients with SARS-CoV-2 infection. Eur Respir J. 2020.

24. Talha B, Dhamoon AS. Ritonavir. [Updated 2019 Nov 28]. In: Stat Pearls [Internet]. Treasure Island (FL): Stat Pearls Publishing; January 2020. Available from: https:// www.ncbi.nlm.nih.gov/books/NBK544312.

25. Chu CM, Cheng VC, Hung IF, Wong MM, Chan KH, Chan KS, Kao RY, et al. Role of lopinavir/ritonavir in the treatment of SARS: initial virological and clinical findings. Thorax. 2004;59(3):252-256.

26. Chen F, Chan KH, Jiang Y, Kao RY, Lu HT, Fan KW, Cheng $\mathrm{VC}$, et al. In vitro susceptibility of 10 clinical isolates of SARS coronavirus to selected antiviral compounds. J Clin Virol. 2004;31(1):69-75.

27. Wu CY, Jan JT, Ma SH, Kuo CJ, Juan HF, Cheng YS, Hsu HH, et al. Small molecules targeting severe acute respiratory syndrome human coronavirus. Proc Natl Acad Sci U S A. 2004;101(27):10012-10017.

28. Zhou ZG, Xie SM, Zhang J, Zheng F, Jiang DX, Li KY, et al. Short-term moderate-dose corticosteroid plus immunoglobulin effectively reverses COVID-19 patients who have failed low-dose therapy. 2020.

29. Zhang Z, Li X, Zhang W, Shi ZL, Zheng Z, Wang T. Clinical features and treatment of 2019-nCov pneumonia patients in wuhan: report of a couple cases. Virol Sin. 2020;35(3):330-336.

30. Chen C, Qi F, Shi K, Li Y, Li J, Chen Y, et al. Thalidomide combined with low-dose glucocorticoid in the treatment of COVID-19 Pneumonia. Clin Transl Med. 2020.

31. Chen C, Huang J, Cheng Z, et al. Favipiravir versus Arbidol for COVID-19: a randomized clinical trial. medRxiv. 2020.

32. Pshenichnaya NY, Bulgakova VA, Lvov NI, Poromov AA, Selkova EP, Grekova AI, Shestakova IV, et al. Clinical efficacy of umifenovir in influenza and ARVI (study ARBITR). Ter Arkh. 2019;91(3):56-63.

33. Hulseberg CE, Feneant L, Szymanska-de Wijs KM, Kessler NP, Nelson EA, Shoemaker CJ, Schmaljohn CS, et al. Arbidol and other low-molecular-weight drugs that inhibit Lassa and Ebola viruses. J Virol. 2019;93(8).

34. Shi L, Xiong H, He J, Deng H, Li Q, Zhong Q, Hou W, et al. Antiviral activity of arbidol against influenza A virus, respiratory syncytial virus, rhinovirus, coxsackie virus and adenovirus in vitro and in vivo. Arch Virol. 2007;152(8):1447-1455.

35. Goldhill DH, Te Velthuis AJW, Fletcher RA, Langat P, Zambon M, Lackenby A, Barclay WS. The mechanism of resistance to favipiravir in influenza. Proc Natl Acad Sci U S A. 2018;115(45):11613-11618.

36. Furuta Y, Takahashi K, Fukuda Y, Kuno M, Kamiyama T, Kozaki K, Nomura N, et al. In vitro and in vivo activities of anti-influenza virus compound T-705. Antimicrob Agents Chemother. 2002;46(4):977-981.

37. Grein J, Ohmagari N, Shin D, Diaz G, Asperges E, Castagna A, Feldt T, et al. Compassionate use of remdesivir for patients with severe COVID-19. N Engl J Med. 2020;382(24):2327-2336.

38. Wang Y, Zhang D, Du G, Du R, Zhao J, Jin Y, Fu S, et al. Remdesivir in adults with severe COVID-19: a randomised, double-blind, placebo-controlled, multicentre trial. Lancet. 2020;395(10236):1569-1578.

39. Beigel JH, Tomashek KM, Dodd LE. Remdesivir for the Treatment of Covid-19 - Preliminary Report. Reply. N Engl J Med. 2020;383.

40. de Wit E, Feldmann F, Cronin J, Jordan R, Okumura A, Thomas T, Scott D, et al. Prophylactic and therapeutic remdesivir (GS-5734) treatment in the rhesus macaque model of MERS-CoV infection. Proc Natl Acad Sci U S A. 2020;117(12):6771-6776.

41. Sheahan TP, Sims AC, Graham RL, Menachery VD, Gralinski LE, Case JB, Leist SR, et al. Broad-spectrum antiviral GS-5734 inhibits both epidemic and zoonotic coronaviruses. Sci Transl Med. 2017;9(396).

42. Sheahan TP, Sims AC, Leist SR, Schafer A, Won J, Brown AJ, Montgomery SA, et al. Comparative therapeutic efficacy of remdesivir and combination lopinavir, ritonavir, and interferon beta against MERS-CoV. Nat Commun. 2020;11(1):222.

43. Mulangu S, Dodd LE, Davey RT, Jr., Tshiani Mbaya O, Proschan M, Mukadi D, Lusakibanza Manzo M, et al. A randomized, controlled trial of Ebola virus disease therapeutics. N Engl J Med. 2019;381(24):2293-2303.

44. European Medicines Agency. Summary on compassionate use: Remdesivir Gilead. April 3, 2020 (https://www. ema.europa.eu/en/documents/other/summary-compacompassionate-use-remdesivir-gilead_en.pdf). 
45. Zhou Q, Wei XS, Xiang X, Wang X, Wang ZH, Chen V, et al. Interferon- $a 2 b$ treatment for COVID-19. MedRxiv. 2020;2020(4).

46. Hung IF, Lung KC, Tso EY, Liu R, Chung TW, Chu MY, $\mathrm{Ng}$ YY, et al. Triple combination of interferon beta-1b, lopinavir-ritonavir, and ribavirin in the treatment of patients admitted to hospital with COVID-19: an open-label, randomised, phase 2 trial. Lancet. 2020;395(10238):16951704.

47. Huang Q, Deng X, Li Y, Sun X, Chen Q, Xie M, Liu S, et al. Clinical characteristics and drug therapies in patients with the common-type coronavirus disease 2019 in Hunan, China. Int J Clin Pharm. 2020;42(3):837-845.

48. Wang BX, Fish EN. Global virus outbreaks: Interferons as 1st responders. Semin Immunol. 2019;43:101300.

49. Kindler E, Thiel V, Weber F. Interaction of SARS and MERS Coronaviruses with the Antiviral Interferon Response. Adv Virus Res. 2016;96:219-243.

50. Yin Y, Wunderink RG. MERS, SARS and other coronaviruses as causes of pneumonia. Respirology. 2018;23(2):130-137.

51. Omrani AS, Saad MM, Baig K, Bahloul A, Abdul-Matin M, Alaidaroos AY, Almakhlafi GA, et al. Ribavirin and interferon alfa-2a for severe Middle East respiratory syndrome coronavirus infection: a retrospective cohort study.
Lancet Infect Dis. 2014;14(11):1090-1095.

52. Rolain JM, Colson P, Raoult D. Recycling of chloroquine and its hydroxyl analogue to face bacterial, fungal and viral infections in the 21 st century. Int J Antimicrob Agents. 2007;30(4):297-308.

53. Okubo K, Isono M, Asano T, Sato A. Lopinavir-ritonavir combination induces endoplasmic reticulum stress and kills urological cancer cells. Anticancer Res. 2019;39(11):5891-5901.

54. Blaising J, Polyak SJ, Pecheur EI. Arbidol as a broadspectrum antiviral: an update. Antiviral Res. 2014;107:8494.

55. Lee N, Allen Chan KC, Hui DS, Ng EK, Wu A, Chiu RW, Wong VW, et al. Effects of early corticosteroid treatment on plasma SARS-associated Coronavirus RNA concentrations in adult patients. J Clin Virol. 2004;31(4):304309.

56. Newfield C. New medical indications for thalidomide and its derivatives new medical indications for thalidomide and its derivatives. The Science Journal of the Lander College of Arts and Sciences. 2018;12.

57. https://www.who.int/emergencies/diseases/novel-coronavirus-2019/global-research-on-novel-coronavirus2019-ncov/solidarity-clinical-trial-for-covid-19-treatments. Last Accessed: May 11, 2020. 